\title{
Subclinical cardiomyopathy in Becker muscular dystrophy
}

\author{
Stephen E Steare, Victor Dubowitz, Avram Benatar
}

\begin{abstract}
Objective-To investigate the prevalence, age distribution, and spectrum of cardiac involvement in a cohort of patients with Becker muscular dystrophy.

Design-A prospective non-invasive study with clinical, electrocardiographic, and echocardiographic assessment.
\end{abstract}

Patients-19 patients (age range 16-41 years) with Becker muscular dystrophy attending the Muscle Clinic at Hammersmith Hospital and 22 healthy controls (age range 22-36 years).

Results-17 patients (89\%) were symptom free; two had exertional dyspnoea. Three had a past history of acute pericarditis. The electrocardiogram was abnormal in 14 patients (74\%). Intraventricular conduction delay or right bundle branch block was present in eight $(42 \%)$. Three $(16 \%)$ had tall $R$ waves $(R / S>1)$ in lead $\mathrm{V} 1$ in the absence of right bundle branch block and eight $(42 \%)$ had $Q$ waves in the lateral and inferolateral leads. The PQ interval was significantly shorter in patients with Becker muscular dystrophy (p < 0.01). Echocardiography showed left ventricular dilatation in seven patients $(37 \%)$ and $12(63 \%)$ had subnormal systolic function caused by global hypokinesia (fractional shortening $<27 \%)$. Six of these patients were under the age of 22 years. Patients with Becker muscular dystrophy had significant reduction of both fractional shortening and corrected mean velocity of circumferential shortening compared with controls. No correlation was found between fractional shortening and age.

The third filling fraction was signifcantly reduced in patients with Becker muscular dystrophy $(p<0.05)$, although other indices of diastolic function (isovolumic relaxation time and $E / A$ ratios) were not significantly different.

Conclusions-Though most patients with Becker muscular dystrophy were symptom free, a high percentage had evidence of a subclinical cardiomyopathy. Electrocardiography showed that the inferolateral and posterior segments of the left ventricle tended to be affected and may show evidence for conduction tissue disease. Echocardiography showed that most patients had left ventricular dilation and global hypokinesia. The severity of left ventricular disease was unrelated to age; some younger patients had severe left ventricular dysfunction. All patients with Becker muscular dystrophy should have echocardiographic assessment of left ventricular function.

\section{(Br Heart J 1992;68:304-8)}

Becker muscular dystrophy is an X-linked recessive neuromuscular disease characterised by progressive muscular weakness. It tends to run a milder course than Duchenne muscular dystrophy with patients remaining ambulant beyond the age of 16 years and usually surviving past the age of 30 years. ${ }^{1}$

Gene mutations at the p21 band of the short arm of the $\mathrm{X}$ chromosome, coding for the protein dystrophin, are found in both Duchenne and Becker muscular dystrophy. In Duchenne muscular dystrophy there is little or no dystrophin whereas in Becker muscular dystrophy it is present in reduced quantity or is of abnormal molecular structure or both. ${ }^{1}$

Cardiac involvement in Becker muscular dystrophy was first recognised in $1966^{2}$ and since then dilated cardiomyopathy has been reported in patients with this disease. ${ }^{34}$ Most patients are free of cardiac symptoms. ${ }^{5}$ A previous prospective study of 14 patients found some evidence of cardiac involvement in $64 \%$; however, none had a dilated cardiomyopathy. ${ }^{6}$ Myocardial involvement in Becker muscular dystrophy seems to be unrelated to the clinical severity or duration of skeletal muscle involvement ${ }^{4}$ and may even precede the onset of muscle weakness. ${ }^{7}$ Evidence of cardiac involvement may be found on electrocardiography. Described abnormalities include intraventricular and right bundle branch blocks, ${ }^{45}$; ST segment abnormalities $^{8}$; $T$ wave abnormalities ${ }^{389}$; and $Q$ waves in the inferior, ${ }^{8}$ lateral, ${ }^{7-9}$ and precordial $^{8}$ leads. The $P Q$ segment (end of $P$ wave to beginning of $Q R S$ ) is shortened and the $\mathrm{QT} / \mathrm{PQ}$ ratio (cardiomyopathic index) is increased in both Becker and Duchenne muscular dystrophy. ${ }^{10}$ These changes are believed to be useful electrocardiographic indicators of early cardiac involvement. ${ }^{10}$ Thallium perfusion studies in patients with Becker muscular dystrophy with dilated cardiomyopathy identified regional perfusion defects in the left ventricle in the absence of coronary artery disease. $^{1112}$

The pathology of the dilated cardiomyopathy in Becker muscular dystrophy consists of diffuse degeneration and fibrosis of both \\ Department of \\ and Neuromuscular \\ Paediatrics and \\ Hospital, London \\ S E Steare \\ Correspondence to \\ Dr Avram Benatar, \\ Wilhelmina \\ 18 March 1992
}


ventricles, especially of the mid-myocardium and the posterolateral portion of the left ventricle. ${ }^{913}$ Necrosis and fibrosis of the conducting tissue have also been reported. ${ }^{13}$

Cardiac disease is likely to be an important determinant of functional capacity and survival in Becker muscular dystrophy because the skeletal and respiratory muscles are less severely affected than in Duchenne muscular dystrophy. Although the dilated cardiomyopathy seen in some patients may represent the end stage of progressive cardiac involvement in Becker muscular dystrophy, there are few data on the prevalence and natural history of cardiac involvement. We report the results of a cross sectional study of cardiac disease in 19 patients.

\section{Patients and methods}

We studied 19 patients attending the Muscle Clinic at Hammersmith Hospital. The research protocol was approved by the ethics committee of the Royal Postgraduate Medical School and informed consent was obtained from each patient. The specific diagnosis of Becker muscular dystrophy, as opposed to Duchenne muscular dystrophy, was made on the basis of the typical clinical features of dystrophy and the ability to walk at the age of 16 years. ${ }^{14}$ The diagnosis was confirmed histologically on muscle biopsy. Sixteen patients had undergone genetic studies and the skeletal muscle dystrophin status had been determined in nine patients by the western blot technique. Patients were divided into three broad clinical groups according to degree of physical disability: (I) minor impairment of motor function, (II) major impairment of motor functionambulant, (III) major impairment of motor function-wheelchair dependent. Comparative data were obtained from 22 healthy volunteers.

A detailed history was taken with particular attention to cardiovascular symptoms and drug history. A full clinical examination was performed. Pulmonary function as assessed by forced vital capacity was measured by standard spirometry and compared with standard tables.

The patients and 22 controls had 12 lead electrocardiograms recorded at speeds of 25 and $50 \mathrm{~mm} / \mathrm{s}$ on a Marquette Mac 12. These were analysed for morphological, rhythm, and conduction abnormalities. The PR and QT intervals and the $P Q$ segment were measured from standard lead II, ${ }^{15}$ and the $P / P Q$ and $Q T /$ $P Q$ ratios were calculated.

Transthoracic echocardiography by $M$ mode, cross sectional Doppler, and colour flow mapping was performed on a Toshiba SSH$160 \mathrm{~A}$ scanner. Both 2.5 and $3.75 \mathrm{mHz}$ probes were used for imaging and Doppler studies. The patients and 19 controls were examined in the left lateral position from standard parasternal and apical views, with simultaneous electrocardiogram and phonocardiogram recordings. All measurements were averaged over 3-5 cardiac cycles. Internal chamber measurements (left ventricular internal dimension in diastole and systole (LVIDd and
LVIDs) and the left atrial dimension) were measured as recommended by the American Society of Echocardiography..$^{16}$ From an apical four chamber view, mitral inflow velocities were assessed with range gated pulsed Doppler echocardiography. The sample volume was positioned parallel to the flow at the site of maximum velocity at the tip of the mitral leaflets. The following were measured: left ventricular ejection time (ET), isovolumic relaxation time, $R R$ interval, peak $E$ and $A$ velocities, and the velocity time integral of total diastolic filling (VTI total) and of the first third of diastolic filling (VTI third).

The following indices of systolic and diastolic function were calculated: shortening fraction (SF = [LVIDd - LVIDs]/LVIDd), corrected mean velocity of circumferential shortening ( $\mathrm{mVcFc}=$ [LVIDd - LVIDs]/ LVIDd $\times$ ETc, where ETc $=\mathrm{ET} /$ square root of the RR interval), E/A velocity ratios (peak velocity $E$ /peak velocity $A$ ), and third filling fraction (VTI total/VTI third).

\section{STATISTICAL ANALYSIS}

The numerical variables are expressed as mean (SD). A two-tailed, unpaired $t$ test was used for statistical analysis. A p value of less than 0.05 was regarded as significant. The $95 \%$ confidence intervals are given.

\section{Results}

\section{CLINICAL PROFILE}

The patients' ages ranged from 16 to 41 years (mean 23, median 22) and those of the 22 controls from 22 to 36 years (mean 27, median 26).

Of the 19 patients, 12 had minor motor impairment (I), four had major impairment but were ambulant (II), and three were wheelchair dependent (III). The dystrophin in all nine patients studied was of a truncated form and present in reduced amounts. Of the 16 patients who underwent genetic studies, 12 had detectable deletions in the Duchenne gene and no deletions were detectable in the remaining four. Patients were divided into three groups according to the site of exon deletions in the Duchenne gene: $A$, exons 13-19; B, exons 9-45; and C, exons 45-49 (table 1).

Seventeen patients were free of cardiac symptoms and two had exertional dyspnoea. In the past, three patients had had acute pericarditis. Except for one patient who was taking atenolol, no one else was receiving regular medication at the time of the study. All were normotensive and none had abnormal cardiac signs.

\section{ELECTROCARDIOGRAPHIC STUDIES}

The electrocardiogram was completely normal in five patients (table 2). All patients were in sinus rhythm apart from one, who in addition had a short run of nodal rhythm. An intraventricular conduction delay including incomplete right bundle branch block was present in six patients and complete right bundle branch block occurred in two others. When patients with incomplete or complete right bundle 
Table 1 Data on patients with Becker muscular dystrophy

\begin{tabular}{|c|c|c|c|c|c|}
\hline Case & $\begin{array}{l}\text { Age } \\
(y r)\end{array}$ & $\begin{array}{l}\text { Functional } \\
\text { grade }\end{array}$ & $\begin{array}{l}\text { Symptoms and } \\
\text { past history }\end{array}$ & Drugs & $\begin{array}{l}\text { Exon } \\
\text { deletions }\end{array}$ \\
\hline \begin{tabular}{r|}
1 \\
2 \\
3 \\
4 \\
5 \\
6 \\
7 \\
8 \\
9 \\
10 \\
11 \\
12 \\
13 \\
14 \\
15 \\
16 \\
17 \\
18 \\
19
\end{tabular} & $\begin{array}{l}17 \\
21 \\
26 \\
19 \\
25 \\
26 \\
16 \\
16 \\
41 \\
16 \\
26 \\
22 \\
22 \\
26 \\
39 \\
25 \\
19 \\
17 \\
16\end{array}$ & $\begin{array}{r}\text { I } \\
\text { I } \\
\text { III } \\
\text { II } \\
\text { II } \\
\text { III } \\
\text { I } \\
\text { I } \\
\text { II } \\
\text { I } \\
\text { I } \\
\text { I } \\
\text { I } \\
\text { I } \\
\text { II } \\
\text { III } \\
\text { I } \\
\text { I } \\
\text { I }\end{array}$ & $\begin{array}{l}\text { None } \\
\text { None } \\
\text { None } \\
\text { None } \\
\text { SOB, P 22 yr } \\
\text { None } \\
\text { SOB } \\
\text { P } 15 \text { yr } \\
\text { None } \\
\text { None } \\
\text { None } \\
\text { None } \\
\text { None } \\
\text { None } \\
\text { None } \\
\text { P 23 yr } \\
\text { None } \\
\text { None } \\
\text { None }\end{array}$ & Atenolol & $\begin{array}{l}45-47(\mathrm{C}) \\
13 \quad(\mathrm{~A}) \\
9-45(\mathrm{~B}) \\
\text { NT } \\
45-48(\mathrm{C}) \\
45 \quad(\mathrm{C}) \\
\text { ND } \\
45-47(\mathrm{C}) \\
\text { NT } \\
\text { NT } \\
46-49(\mathrm{C}) \\
45-47(\mathrm{C}) \\
\text { ND } \\
45-47(\mathrm{C}) \\
13-19(\mathrm{~A}) \\
19-44(\mathrm{~B}) \\
\text { ND } \\
\text { ND } \\
48 \quad \text { (C) }\end{array}$ \\
\hline
\end{tabular}

SOB, shortness of breath; P, pericarditis; I, minor motor impairment; II, major motor impairment-ambulant; III, wheelchair dependent; Exon deletion groups: A, 13-19; B, 9-45; C, 45-49; ND, no deletion detected; NT, not tested.

Table 2 Spirometry, electrocardiography, and echocardiography in patients with Becker muscular dystrophy

\begin{tabular}{|c|c|c|c|c|c|c|}
\hline Case & $\begin{array}{l}F V C \\
(\%)\end{array}$ & Electrocardiogram & $\begin{array}{l}Q T c \\
\text { (ms) }\end{array}$ & $Q T / P Q$ & $\underset{(\mathrm{mm})}{\text { LVIDd }}$ & $\begin{array}{l}F S \\
(\%)\end{array}$ \\
\hline $\begin{array}{r}1 \\
2 \\
3 \\
4 \\
5 \\
6 \\
7 \\
8 \\
9 \\
10 \\
11 \\
12 \\
13 \\
14 \\
15 \\
16 \\
17 \\
18 \\
19\end{array}$ & $\begin{array}{r}96 \\
93 \\
42 \\
89 \\
77 \\
42 \\
71 \\
\text { NT } \\
78 \\
65 \\
80 \\
98 \\
53 \\
98 \\
\text { NT } \\
\text { NT } \\
100 \\
82 \\
82\end{array}$ & $\begin{array}{l}\text { IRBBB } \\
\text { RBBB } \\
\text { IVCD } \\
\text { Run nodal rhythm, IVCD } \\
\text { Normal } \\
\text { Tall R V1, Lat Q \& T abn } \\
\text { Tall R V1, Lat } Q \\
\text { Tall R V1, Lat Q \& R loss } \\
\text { Reduced R V2-V3 } \\
\text { LAH, IRBBB, Inf-lat T abn } \\
\text { LVH } \\
\text { Normal } \\
\text { IVCD, Lat Q \& T abn } \\
\text { Normal } \\
\text { Normal } \\
\text { Normal } \\
\text { Inf-lat } Q \\
\text { IVCD, Lat } Q \\
\text { RBBB, LVH, Lat } Q\end{array}$ & $\begin{array}{l}399 \\
408 \\
423 \\
410 \\
404 \\
441 \\
409 \\
432 \\
442 \\
456 \\
430 \\
391 \\
444 \\
402 \\
412 \\
386 \\
389 \\
388 \\
403\end{array}$ & $\begin{array}{r}10 \cdot 70 \\
10 \cdot 20 \\
7 \cdot 36 \\
10 \cdot 01 \\
8 \cdot 80 \\
8 \cdot 60 \\
8 \cdot 25 \\
7 \cdot 36 \\
10 \cdot 20 \\
10 \cdot 00 \\
10 \cdot 00 \\
11 \cdot 80 \\
9 \cdot 75 \\
18 \cdot 00 \\
18 \cdot 20 \\
5 \cdot 25 \\
12 \cdot 66 \\
13 \cdot 86 \\
9 \cdot 30\end{array}$ & $\begin{array}{l}51 \\
52 \\
42 \\
62 \\
47 \\
66 \\
56 \\
48 \\
58 \\
53 \\
51 \\
55 \\
58 \\
55 \\
48 \\
46 \\
46 \\
50 \\
52\end{array}$ & $\begin{array}{l}24 \\
21 \\
23 \\
15 \\
27 \\
13 \\
22 \\
26 \\
21 \\
26 \\
27 \\
29 \\
15 \\
16 \\
23 \\
32 \\
28 \\
29 \\
29\end{array}$ \\
\hline
\end{tabular}

FVC, forced vital capacity (\% predicted); LVIDd, left ventricular internal dimension in diastole; FS\%, percentage left ventricular fractional shortening, IRBBB, incomplete right bundle branch block; RBBB, complete right bundle branch block; IVCD, intraventricular conduction delay; Lat, lateral; Inf-lat, inferolateral; LAH, left anterior hemiblock; LVH, left ventricular hypertrophy; NT, not tested.

branch block were excluded, pathologically tall $R$ waves $(R / S$ in $V 1<1)$ were present in three patients. In a further six, the $R / S$ ratio in $V 1$ $>0.5 \mathrm{mV}$ whereas the $\mathrm{R} / \mathrm{S} \mathrm{V} 1$ ratio in controls was always $<0.5 \mathrm{mV}$. In four patients $Q$ waves were seen in the lateral leads (amplitude $>0.5 \mathrm{mV}$ in leads I and aVL) and in two of these $Q$ waves were also present in the inferior leads (amplitude $>0.3 \mathrm{mV}$ in leads II and aVF). A further three patients had small $Q$ waves $(0.2-0.5 \mathrm{mV})$ in the lateral leads. $Q$ wave duration was between $20 \mathrm{~ms}$ and $40 \mathrm{~ms}$ and in only one of the seven was associated with $R$ wave loss. One other patient had $R$ wave voltage reduction in the anterior chest leads. $T$ wave abnormalities were seen in three patients-associated with lateral $Q$ waves in two and as a sole abnormality in the inferolateral leads in one other. Two patients had electrical voltage criteria for left ventricular hypertrophy without a strain pattern.

There was no significant difference in the resting heart rates or the $P$ wave duration between the two groups. The $P R$ and $P Q$ intervals were significantly shorter and the $Q T C$ intervals were significantly longer in patients with Becker muscular dystrophy (table 3), however. In four patients QTc was $>440 \mathrm{~ms}$, and in two of these patients had intraventricular conduction delays.

Since the effect of heart rate on the $P / P Q$ and $\mathrm{QT} / \mathrm{PQ}$ ratios has not been clearly established we compared the $P / P Q$ and $Q T / P Q$ ratios in two heart rate bands (table 4). A patient with a resting heart rate of 100 beats $/ \mathrm{min}$ was excluded from analysis. Significant differences in both these ratios were found between the Becker and control groups even when heart rates were matched in this way. No correlation was found between the $\mathrm{QT} / \mathrm{PQ}$ or $\mathrm{P} / \mathrm{PQ}$ ratios and the genetic deletion group or with left ventricular function as determined by fractional shortening.

\section{ECHOCARDIOGRAPHIC STUDIES}

No valve abnormality was detected in any individual. Left ventricular dilatation (LVIDd $>95 \%$ of the predicted upper limit for body weight) was seen in seven of the 19 patients (table 2). None of the patients had left atrial enlargement. Delayed and reduced septal wall motion was noted in two patients-one with complete right bundle branch block and another with incomplete right bundle branch block. Regional wall motion abnormalities were not seen in any of the other subjects.

Estimates of systolic function were subnormal in 12 patients (table 2). As a group, the

Table 3 Electrocardiographic values in patients with Becker muscular dystrophy and controls

\begin{tabular}{|c|c|c|c|c|c|}
\hline & $\begin{array}{l}H R \\
\left(\min ^{-1}\right)\end{array}$ & $\begin{array}{l}P R \\
(m s)\end{array}$ & $\begin{array}{l}P \\
(m s)\end{array}$ & $\underset{(m s)}{P Q}$ & $\underset{(m s)}{Q T c}$ \\
\hline $\begin{array}{l}\text { Becker }(=19) \\
\text { Controls }(n=22) \\
\text { CI }\end{array}$ & $\begin{array}{c}73.1(11.8) \\
67.6(11.6) \\
-1.9 \text { to } 12.9\end{array}$ & $\begin{array}{c}134 \cdot 1(18.5) \dagger \\
156 \cdot 0(21 \cdot 1) \\
-34.5 \text { to }-9.4\end{array}$ & $\begin{array}{r}92.5(13.9) \\
97.6(14.2) \\
-14.0 \text { to } 3.8\end{array}$ & $\begin{array}{c}40 \cdot 0(12 \cdot 0) \dagger \\
58 \cdot 4(16 \cdot 4) \\
-27 \cdot 6 \text { to }-9 \cdot 2\end{array}$ & $\begin{array}{c}414.2(21.2)^{\star} \\
400 \cdot 8(18.2) \\
0.9 \text { to } 25 \cdot 8\end{array}$ \\
\hline
\end{tabular}

HR, heart rate; PR, PR interval; P, P wave duration; PQ, PQ interval; $Q T c, Q T c$ interval; $C I, 95 \%$ confidence intervals ${ }^{\star} \mathrm{p}<0.05,+\mathrm{p}<0.01$.

Table 4 Comparison of $P / P Q$ and $Q T / P Q$ ratios in two heart rate bans in patients with Becker muscular dystrophy and controls

\begin{tabular}{|c|c|c|c|c|c|c|}
\hline & \multicolumn{3}{|c|}{ HR 50-70 beats $/ \mathrm{min}$} & \multicolumn{3}{|c|}{ HR $71-90$ beats $/ \mathrm{min}$} \\
\hline & $n$ & $P / P Q$ & $Q T / P Q$ & $n$ & $P / P Q$ & $Q T / P Q$ \\
\hline $\begin{array}{l}\text { Becker } \\
\text { Controls } \\
\text { CI }\end{array}$ & $\begin{array}{l}7 \\
9\end{array}$ & $\begin{array}{l}2.56(0.86){ }^{\star} \\
1.67(0.44) \\
0.18 \text { to } 1.6\end{array}$ & $\begin{array}{c}10.40(2.71)^{\star} \\
7.38(1.84) \\
0.6 \text { to } 5.45\end{array}$ & $\begin{array}{l}11 \\
11\end{array}$ & $\begin{array}{l}2.75(1.12)^{\star} \\
1.83(0.64) \\
0.11 \text { to } 1.73\end{array}$ & $\begin{array}{c}10.82(3.82) \dagger \\
6.72(1.59) \\
1.5 \text { to } 6.7\end{array}$ \\
\hline
\end{tabular}


Table 5 Echocardiographic values in patients with Becker muscular dystrophy and controls

\begin{tabular}{|c|c|c|c|c|c|}
\hline & $\begin{array}{l}F S \\
(\%)\end{array}$ & $\begin{array}{l}m V c F c \\
(c m / s)\end{array}$ & $\begin{array}{l}I R T \\
(m s)\end{array}$ & $E / A$ & $\begin{array}{l}\text { Third filling } \\
\text { fraction }\end{array}$ \\
\hline $\begin{array}{l}\text { Becker }(=19) \\
\text { Controls }(\mathrm{n}=19) \\
\text { CI }\end{array}$ & $\begin{array}{c}23.47(5.51) \dagger \\
32.89(4.78) \\
-12.8 \text { to }-6.0\end{array}$ & $\begin{array}{c}0.78(0.18) \dagger \\
1.07(0.13) \\
-0.4 \text { to }-0.19\end{array}$ & $\begin{array}{c}54 \cdot 20(11 \cdot 1) \\
50.79(8 \cdot 82) \\
-3.2 \text { to } 10\end{array}$ & $\begin{array}{c}2.16(0.94) \\
2.10(0.33) \\
-0.42 \text { to } 0.53\end{array}$ & $\begin{array}{c}0.58(0.13)^{\star} \\
0.67(0.10) \\
-0.17 \text { to }-0.01\end{array}$ \\
\hline
\end{tabular}

patients with Becker muscular dystrophy had a significantly smaller shortening fraction and corrected mean velocity of circumferential shortening than the controls (table 5).

Fractional shortening did not correlate with pulmonary function, age, the severity of skeletal muscle involvement, or the patients' gene deletion site.

The third filling fraction was significantly reduced in the Becker group, although other indices of diastolic function (isovolumic relaxation time and E/A velocity ratio) were not significantly different from values in the controls (table 5).

\section{Discussion}

The prevalence, spectrum, and timing of onset of cardiac disease in Becker muscular dystrophy has received little attention despite the recognised association with a dilated cardiomyopathy. ${ }^{2-4}$ We found a surprisingly high frequency of electrocardiographic and echocardiographic evidence of important subclinical cardiac involvement in this condition.

The electrocardiogram was abnormal in $74 \%$ of patients, and this may be an early indicator of cardiac disease. Non-specific changes, in particular intraventricular and right bundle branch block, were frequent $(42 \%)$. Others have reported these changes in patients with Becker muscular dystrophy ${ }^{45}$ and they may reflect damage to the intraventricular conduction system, which is known to occur in patients with a dilated cardiomyopathy. ${ }^{213}$ Such involvement may even lead to complete heart block $\hat{k}^{2}$ and should not be underestimated. Ambulatory electrocardiographic monitoring may provide additional information about disease of the conducting tissue in these patients. Specific changes were common (47\%) and most often took the form of a "pseudoinfarction" pattern, comprising of narrow $Q$ waves without associated $R$ wave loss in the inferolateral leads $(42 \%)$ and/or tall $R$ waves in the right precordial leads (16\%). These two types of abnormalities have been reported in patients with Duchenne muscular dystrophy ${ }^{17} 18$ and in patients with Becker muscular dystrophy. ${ }^{379}$ In Duchenne muscular dystrophy the histological basis for these changes is diffuse myocardial fibrosis with a tendency for early involvement of the posterobasal and adjacent free wall of the left ventricle. ${ }^{1719} \mathrm{It}$ is likely that the electrocardiographic abnormalities see in Becker muscular dystrophy reflect a similar underlying disease process.

In three patients with regional electrocardiographic changes there was no demonstrable left ventricular dysfunction on echo- cardiography. Furthermore, in all nine patients with these abnormalities corresponding regional wall motion abnormalities were not detected. Thus the electrocardiogram may be very sensitive in detecting early ventricular pathology.

QTc prolongation was present in $16 \%$ of patients and in all four patients was associated with appreciable left ventricular dysfunction. Thus it may provide additional evidence of left ventricular disease in patients with Becker muscular dystrophy.

Nigro et al ${ }^{10}$ emphasised the value of the QT/ $P Q$ ratio, the "cardiomyopathic index", as an early marker of cardiac involvement in the progressive X-linked muscular dystrophies. Our data confirm that this index and the $P / P Q$ ratio are both increased in patients with Becker muscular dystrophy. The PQ segment was significantly shorter in Becker patients and accounts for the higher $Q T / P Q$ and $P / P Q$ ratios. Shortening of the $P Q$ interval and an increase of the $P / P Q$ ratio are associated with either left atrial enlargement ${ }^{20}$ or left atrial fibrosis. ${ }^{15}$ Because the left atrial dimension was normal, in all of the patients studied, these electrocardiographic changes are likely to reflect left atrial fibrosis. No association was found between the $Q T / P Q$ ratio and left ventricular fractional shortening. Whereas the $\mathrm{QT} / \mathrm{PQ}$ ratio may provide a useful indicator of atrial disease it should not be used alone to gauge the severity of overall cardiac involvement.

Echocardiography showed that an unexpectedly high percentage of patients $(67 \%)$ had subnormal left ventricular function. In addition $31 \%$ had left ventricular dilatation. The severity of left ventricular disease was unrelated to age, and, more importantly, severe left ventricular impairment occurred in some patients in their teens and early twenties. We predict that such young patients, who often have only slight impairment of skeletal muscle, will develop congestive cardiac failure in the near future. There are considerable therapeutic implications in the management of such patients both before and after onset of cardiac symptoms. The possible benefits from the early use of vasodilator therapy in these patients will require proper evluation. Cardiac transplantation may also be an effective treatment for those patients with cardiac failure refractory to medical treatment, who would otherwise have a reasonable life expectancy. ${ }^{413}$

Left ventricular dysfunction is common in Duchenne muscular dystrophy ${ }^{21} 22$ and it does not seem to be directly associated with respiratory impairment. ${ }^{22} \mathrm{We}$ also found this to be true of Becker muscular dystrophy. It seems 
that in Becker muscular dystrophy the onset and progression of cardiac disease bear no relation to involvement of the skeletal and thoracic cage muscles. In addition, we did not find an association between left ventricular function and the underlying deletion on the Duchenne gene. Currently there is no evidence that the type of gene deletion is predictive of the extent of cardiac involvement.

We used mitral inflow velocities to estimate the diastolic properties of the left ventricle. Abnormalities of diastolic function were expected in those patients with a cardiomyopathy because they have extensive and diffuse ventricular fibrosis. ${ }^{213}$ The third filling fraction was the only index of diastolic function found to be significantly different from indices in the controls. Echocardiographic evaluation of diastolic function may be influenced by atrial disease and compliance, ${ }_{2}^{23}$ and this may account for the discrepancy in the results. We cannot draw conclusions about ventricular compliance from this study.

Despite having considerable and often severe cardiac disease, most patients were free of cardiac symptoms. It has previously been suggested that the physical limitations imposed by the skeletal muscle disease in Duchenne muscular dystrophy protects the individual against the demands that may lead to cardiac symptoms. ${ }^{21}$ Although some of the patients with Becker muscular dystrophy experienced severe physical limitations, most were ambulant and active and yet they remained symptom free. Dyspnoea may be the earliest symptom of cardiac failure, but it may also be caused by respiratory impairment in patients with Becker muscular dystrophy. A detailed assessment of both systems is required to elucidate the aetiology of this symptom.

Acute pericarditis occurred in $16 \%$, a frequency that was greater than expected. In only one of the three patients was there detectable myocardial involvement. Though acute pericarditis may be more frequent in Becker muscular dystrophy, it is not clear whether it is part of the spectrum of cardiac involvement in this disease. In conclusion, this study has shown that whereas most patients with Becker muscular dystrophy are free of cardiac symptoms, subclinical cardiac disease is very common: it may be severe and it may be present in adolescence. The electrocardiogram may show that the left ventricle is affected, with early evidence of abnormalities of the posterobasal and lateral wall, left atrial fibrosis, and conduction tissue disease. The echocardiogram is essential in the assessment of left ventricular size and function. Careful follow up is required to determine the rate of progression of cardiac disease in these patients.

We thank Professor M Bobrow and colleagues (Guy's Hospital Medical School, London) and Dr P N Strong and Dr Caroline Sewry (Neuromuscular Unit Laboratories, Royal Postgraduate Medical School) for the deletion studies and dystrophin analyses, respectively.

1 Darras BT. Molecular genetics of Duchenne and Becker muscular dystrophy. J Pediatr 1990;117:1-15.

2 Perloff JK, Deleon AC, O'Doherty D. The cardiomyopathy of progressive muscular dystrophy. Circulation 1966; 33:625-58.

3 Katiyar BC, Misra S, Somani PN, Chaterii AM. Congestive cardiomyopathy in a family of Beckers X-linked muscular dystrophy. Postgrad Med J 1977;53:12-5.

4 Sakata C, Yamada H, Sunohara N, Arahara K, Nonaka I. Cardiomyopathy in Becker muscular dystrophy. Rinsho Shinkeigaku 1990;30:952-5.

5 Emery AEH, Skinner R. Clinical studies in benign (Becker type) X-linked muscular dystrophy. Clin Genet 1976 10:189-201.

6 Nigro G, Limongelli FM, Giugliano M-AM, Politano L Passamano L, Stefanelli S. Prospective study of X-linked progressive muscular dystrophy in Campania. Muscle Nerve 1093;6:253-62.

7 Sakata C, Sunohara N, Nonaka I, Arahata K, Sugita H. A case of Becker muscular dystrophy presenting as cardiac failure as an initial symptom. Rinsho Shinkeigaku 1990 failure as an

8 Markand ON, North RR, D'Agostino AN, Daly DD Benign sex linked muscular dystrophy. Neurology 1969 ; 19:617-33.

9 Ueda K, Okada R, Matsuo $H$, Hamurni K, Yasuda $H$ Tsyuki $H$, Ueda $H$. Myocardial involvement in benign Duchenne type of muscular dystrophy. Jap Heart 1970;11:26-35.

10 Nigro G, Comi LI, Politano L, et al. Electrocardiographic evaluation of the $P$ type stage of dystrophic cardiomyopathy. Cardiomyology 1984;3:45-58.

11 Levin RN, Nahara KA. Right axis deviation and anterio wall thallium-201 defect in Becker's muscular dystrophy. Am J Cardiol 1985;56:203-

12 Lazzeroni E, Favaro L, Botti G. Dilated cardiomyopathy with regional myocardial hypoperfusion in Becker's muswith regional myocardial hypoperfusion in Beck

13 Donofrio PD, Challa VR, Hackshaw BT, Mills SA, Cordell $R$. Cardiac transplant in a patient with muscular dystrophy and cardiomyopathy. Arch Neurol 1989;46:705-7.

14 Hodgson S, Hart K, Abbs S, Heckmatt J, Rodillo E Bowbrow M, Dubowitz V. Correlation of clinical and deletion data in Duchenne and Becker muscular dystrophy. J Med Genet 1989;26:682-93.

15 Scott CC, Leier CV, Kilman JW, Vasko JS, Unverferth DV. The effect of left atrial histology and dimension on $P$ wave morphology. J Electrocardiol 1983;16:363-6.

16 Sahn DJ, DeMaria A, Kisslo J, Weyman A. Recommendations regarding quantation in $\mathbf{M}$ mode echocardiography: Result of a survey of echocardiographic measurements. Circulation 1978;58:1072-107.

17 Perloff JK, Roberts WC, Deleon AC, O'Doherty D. The distinctive electrocardiogram of Duchenne progressive muscular dystrophy. An electrocardiographic pathological correlative study. Am J Med 1967;42:179-88.

18 Sanyal SK, Johnson WW, Thapar MK, Pitner SE. A ultrastructural basis for electrocardiographic alteration associated with Duchenne's progressive muscular dystrophy. Circulation 1978;57:1122-48.

19 Frankel KA, Rosser RJ. The pathology of the heart in progressive muscular dystrophy; epimyocardial fibrosis. Hum Pathol 1976;7:375-86.

20 Macruz R, Perloff JK, Case RB. A method for the electrographic recognition of atrial enlargement. Circulation 1958;17:882-9.

21 Danilowicz D, Rutkowski M, Myung D, Schively D. Echocardiography in Duchenne muscular dystrophy. Muscle Nerve 1980;3:298-303.

22 Stewart CA, Gilgoff I, Baydur A, Prentice W, Applebaum D. Gated radionucleotide ventriculography in the evluation of cardiac function in Duchenne muscular dystrophy. Chest 1988;94:1245-8.

23 Shapiro SM, Bersohn MM, Laks MM. In search of the holy grail: the study of diastolic ventricular function by the use of doppler echocardiography. J Am Coll Cardiol 1991; 17:1517-9. 